\title{
Comparación entre uso y no uso de antibióticos profilácticos en pancreatitis aguda grave. Ensayo clínico aleatorizado. Reporte preliminar
}

\author{
Héctor Losada M. ${ }^{1,2,3}$, Sonia Curitol-Sánchez ${ }^{1}$, Andrés Troncoso-Trujillo ${ }^{1,2}$ y \\ Pablo San Martín-Ferrada ${ }^{1}$
}

\section{Comparison between use and non-use of prophylactic antibiotics in severe acute pancreatitis. Randomized clinical trial. Preliminary report}

Introduction: Acute pancreatitis is a common disease in the country, with a mortality rate of $10 \%-30 \%$. The prophylactic administration of antibiotics has been part of the treatment of severe acute pancreatitis (SAP), due to the theoretical prevention of infectious complications and mortality reduction. However, the available scientific evidence is controversial. Objective: To demonstrate that prophylactic antibiotics do not reduce local and/or systemic complications, critical patient unit (CPU) requirement, or mortality in SAP. We define SAP as APACHE II $\geq 8$ or PCR $\geq 150^{\circ}$ or multiorgan failure. Material and Method: Randomized clinical trial, with simple randomization by electronic table (use or non-use of prophylactic antibiotics) of patients with SAP. In the group that used prophylactic antibiotics, ciprofloxacin and metronidazole were used for 7 days. Results: $\mathrm{n}=71$, two randomized groups; Group $1(\mathrm{n}=35)$, without the use of prophylactic antibiotics, and group $2(n=36)$ with the use of antibiotic prophylaxis. 12 patients $(16 \%)$ required CPU; 6 patients from group 1 , and 6 from group $2(p=0.957)$. Seven patients $(9.8 \%)$ had some type of complication, 3 in group 1 and 4 in group $2(p=0.516)$. The average hospital stay was 18.2 \pm 9.5 days in group 1 , and $22.6 \pm 29.2$ days in group $2(\mathrm{p}=0.495)$. Mortality: 1 patient $(1.41 \%)$ in group $2(p=0.493)$. Conclusion: In this preliminary report, the use of prophylactic antibiotics in SAP was not shown to reduce complications, need for bed in CPU, or mortality.

Key words: acute pancreatitis; antibiotic prophylaxis; randomized clinical trial.

\section{Resumen}

Introducción: La pancreatitis aguda es una enfermedad frecuente en el país, con una tasa de mortalidad de $10 \%-30 \%$. La administración profiláctica de antibióticos ha sido parte del tratamiento de pancreatitis aguda grave (PAG), por la teórica prevención de complicaciones infecciosas y reducción de mortalidad. Sin embargo, la evidencia científica disponible es controversial. Objetivo: Demostrar que los antibióticos profilácticos no disminuyen las complicaciones locales y/o sistémicas, requerimiento de Unidad de Paciente Crítico (UPC), ni mortalidad en PAG. Definimos PAG como APACHE II $\geq 8$ o PCR $\geq 150$ o falla multiorgánica. Material y Método: Ensayo clínico aleatorizado, con aleatorización simple mediante tabla electrónica (uso o no uso de antibióticos profilácticos) de pacientes con PAG. En el grupo que usó antibióticos profilácticos se utilizó ciprofloxacino y metronidazol por 7 días. El resto del manejo no tuvo variación. Resultados: $n=71$, dos grupos aleatorizados; Grupo $1(n=35)$, sin uso de antibióticos profilácticos, y grupo $2(\mathrm{n}=36)$ con uso de profilaxis antibiótica. 12 pacientes $(16 \%)$ requirieron UPC; 6 pacientes del grupo 1 , y 6 del grupo $2(\mathrm{p}=0,957)$. Siete pacientes $(9,8 \%)$ tuvieron algún tipo de complicación, 3 en el grupo 1 y 4 en el grupo $2(\mathrm{p}=0,516)$. El promedio de estancia hospitalaria fue $18,2 \pm$ 9,5 días en el grupo 1 , y $22,6 \pm 29.2$ días en el grupo $2(\mathrm{p}=0,495)$. Mortalidad: 1 paciente $(1,41 \%)$ en el grupo $2(p=0,493)$. Conclusión: En este reporte preliminar, el uso de antibióticos profilácticos en PAG no mostró reducir las complicaciones, necesidad de cama en UPC, ni la mortalidad.

Palabras clave: pancreatitis aguda; profilaxis antibiótica; ensayo clínico aleatorizado
Equipo de Cirugía

Hepato-Bilio-Pancreática. Departamento de Cirugía, Traumatología y Anestesiología. Universidad de La Frontera. Temuco, Chile. ${ }^{2} E q u i p o$ de Cirugía HepatoBilio-Pancreática. Hospital Hernán Henríquez Aravena. Temuco, Chile.

${ }^{3}$ Equipo de Cirugía HepatoBilio-Pancreática. Clínica Alemana de Temuco. Temuco, Chile.

Financiamiento: Proyecto DIUFRO N DI17-0062.

Publicación: este estudio se presentó en el 3er Congreso Latinoamericano de Cirugía Pancreática y Biliar Hepato (septiembre de 2017 Viña del mar, Chile) y en el $90^{\circ}$ Congreso Chileno e Internacional de Cirugía (noviembre de 2017, Pucón, Chile)

Recibido el 10 de junio de 2019 y aceptado para publicación el 29 de julio de 2019

Correspondencia a:

Héctor Losada M. hector.losada@ufrontera.cl 


\section{Introducción}

La pancreatitis aguda (PA) es una patología frecuente, corresponde a una de las principales causas de hospitalización entre los pacientes con dolor abdominal ${ }^{1}$. Alrededor de un $80 \%$ de los pacientes se recupera totalmente en 1 semana $^{2}$, ya que se desarrolla en su mayoría como enfermedad no complicada, sin eventos infecciosos, y sin requerir tratamiento intensivo. Sin embargo, un $20 \%$ de los pacientes presentan complicaciones locales o sistémicas, con una tasa de mortalidad de 10\%-30\% ${ }^{3,4}$. El Consenso de Atlanta define como pancreatitis aguda grave (PAG) aquella asociada morfológicamente a necrosis extendida del tejido pancreático $(>30 \%)$, infección debida a necrosis o formación de absceso, y/o presencia de necrosis retroperitoneal de tejido extra-pancreático. Más importante aún, la PAG se identifica por la presencia de complicaciones orgánicas sistémicas (falla pulmonar, renal o hepática) y disfunción cardiopulmonar (shock) $)^{5,6}$.

La PAG es el único modelo de profilaxis antibiótica prolongada. Existe un gran número de revisiones sistemáticas de Ensayos Clínicos Aleatorizados (ECA) que demuestran la efectividad clínica de los antibióticos profilácticos en pancreatitis, que reducen la mortalidad y la incidencia de infección ${ }^{7-9}$, mientras que otras revisiones no han encontrado un beneficio clínico significativo del uso de antibióticos profilácticos ${ }^{10-12}$.

Si hablamos de predictores de gravedad, los criterios del Consenso de Atlanta han sido modificados en la guía clínica del Reino Unido, y estas modificaciones consisten en considerar como PAG a los pacientes que tengan un score APACHE II $>8$ o PCR $>150^{13}$.

Durante las últimas décadas, la administración profiláctica de antibióticos ha sido parte del tratamiento de PAG en nuestro medio, para la teórica prevención de complicaciones infecciosas y reducción de mortalidad $^{13}$, sin embargo, la evidencia científica disponible actualmente es controversial; razón por la cual hemos decidido realizar un ensayo clínico aleatorio (ECA) para comparar el uso y no uso de antibióticos profilácticos, en términos de complicaciones infecciosas locales, complicaciones infecciosas sistémicas, requerimiento de ingreso a Unidad de Paciente Crítico (UPC) y en términos de mortalidad.

\section{Material y Método}

\section{Diseño}

Ensayo clínico aleatorizado, con aleatorización simple (uso o no uso de antibióticos profilácticos).
La asignación aleatoria se realizó mediante tabla computacional. Este es un reporte preliminar que cuenta con el $25 \%$ de la muestra total estimada.

Población: Pacientes con PAG evaluados y tratados por el equipo de cirugía biliopancreática del HHHA entre el 1 de abril de 2016 y el 30 de junio de 2017.

\section{Criterios de inclusión}

Todos los pacientes con PAG que ingresaron al Hospital Dr. Hernán Henríquez Aravena y fueron tratados por el equipo de cirugía hepatobiliar.

\section{Criterios de exclusión}

Pancreatitis agudas catalogadas como leve (PAL).

Paciente que inició antibiótico por sospecha de infección, ya que el concepto de tratamiento de infección es distinto al concepto de profilaxis.

Paciente que tuvo algún otro esquema antibiótico por otro foco infeccioso no pancreático.

\section{Maniobra}

Los pacientes que ingresaron con diagnóstico de PA se clasificaron según el score de gravedad APACHE II y según valor de PCR. Los pacientes con APACHE II $\geq 8$, o PCR $\geq 150$ (valor normal $<10$ $\mathrm{mg} / \mathrm{dl}$ ) o disfunción multiorgánica, se clasificaron como PAG.

Una vez confirmado el diagnóstico de PAG se hizo la aleatorización mediante tabla computacional simple por los coordinadores del trabajo de investigación.

En el grupo de uso de antibióticos profilácticos se utilizó ciprofloxacino y metronidazol. Se usó idealmente la vía oral o por sonda nasoenteral: 500 $\mathrm{mg}$ cada $12 \mathrm{~h}$ de ciprofloxacino y $500 \mathrm{mg}$ cada $8 \mathrm{~h}$ de metronidazol. Se reservó el uso de ciprofloxacino endovenoso para pacientes con incapacidad de tolerar el uso de antibióticos por vía oral y/o sonda nasoenteral, por ejemplo, en los pacientes con íleo; la dosis utilizada de antibióticos endovenosos fue ciprofloxacino $400 \mathrm{mg}$ cada $12 \mathrm{~h}$ y metronidazol 500 mg cada $8 \mathrm{~h}$. La duración de la profilaxis antibiótica se dejó a criterio del equipo de cirugía biliopancreática, pero empíricamente está definido como 7 días.

El resto del tratamiento (soporte nutricional, traslado a UPC, realización de imágenes de control, realización de cirugía o procedimientos) no tuvo variación entre los grupos.

\section{Definición de variables}

Complicaciones: medida como variable dicotómica, en términos de presencia de complicación o no. 
Complicaciones infecciosas locales: en caso de sospecha clínica y/o radiológica de infección peripancreática que requieran tratamiento antibiótico empírico de amplio espectro.

Sepsis de fuente no pancreática: episodios de sepsis de origen no pancreático documentado por imágenes o cultivos que obliguen a procedimientos terapéuticos o a iniciar o cambiar esquema antibiótico.

Requerimiento de ingreso a Unidad de Paciente Crítico (UPC): medida como variable dicotómica (presente o ausente) cuando la causa de ingreso sea disfunción orgánica o sepsis.

Estancia hospitalaria: medida como variable continua, en días.

Mortalidad: medida como variable dicotómica (presente o ausente).

\section{Cálculo del tamaño de la muestra}

Se realizó en el programa EPI INFO con base en el metaanálisis japonés de Ukai et al. ${ }^{9}$ que demostró que la tasa de infección de necrosis en el grupo que no usó antibióticos fue de $25 \%$, mostrando una reducción de $10 \%$ en el grupo que usó antibióticos. Utilizando un nivel de significancia estadística de $95 \%$ y un poder de $80 \%$, el tamaño de muestra es de 140 pacientes por cada grupo, con total de 280 pacientes en total.

\section{Herramientas estadísticas}

La base de datos para el estudio fue confeccionada en Excel y el análisis se efectuó con el programa STATA 10.0. Se utilizaron herramientas de estadística descriptiva con medidas de tendencia central y dispersión; herramientas de estadística analítica con chi 2 o test exacto de Fisher para variables dicotómicas y t-test para variables continuas. La magnitud de asociación fue medida en odds ratio y riesgo absoluto.

\section{Aspectos éticos}

El protocolo fue aprobado por el comité de ética científica del Servicio de Salud Araucanía Sur (Chi- le) y por el grupo de investigación del Departamento de Cirugía, Traumatología y Anestesiología de la Universidad de La Frontera.

\section{Financiamiento}

El proyecto fue financiado por proyecto DIUFRO $\mathrm{N}^{\circ}$ DI17-0062.

\section{Resultados}

El estudio está compuesto por 71 pacientes (25\% de la muestra total), divididos en dos grupos aleatorizados; grupo 1 (sin uso de antibióticos profilácticos) compuesto por 35 pacientes, y grupo 2 (con uso de profilaxis antibiótica) compuesto por 36 pacientes.

La edad promedio del grupo total $(n=71)$ fue de $59,6 \pm 18,4$ años; la edad promedio del grupo 1 fue de 56,5 $\pm 18,9$ años y del grupo $262,6 \pm 17,6$ años (Tabla 1).

La distribución por género mostró un predominio del género femenino en el grupo total con un 57\% $(n=41)$; el grupo 1 tuvo un $65 \%(n=23)$ de pacientes de género femenino, y el grupo $250 \%(\mathrm{n}=18)$ (Tabla 1).

La principal etiología fue litiásica, siendo esta la etiología del 94,2\% en el grupo 1 y del 94,4\% en el grupo 2 (Tabla 1).

El promedio de amilasa $(\mathrm{u} / \mathrm{L})$ de ingreso del total de pacientes fue de $1.123 \pm 1.043$; en el grupo 1 fue de $1.466 \pm 1.133$, y en el grupo 2 fue de $981 \pm 896$. El promedio de lipasa $(\mathrm{u} / \mathrm{L})$ de ingreso del total de pacientes fue de 2.512 \pm 2.893 ; en el grupo A fue de $3.393 \pm 3.578$, y en el grupo B fue de $1.632 \pm 1.611$.

El promedio de PCR de ingreso del total de pacientes fue de $127 \pm 110$,4; en el grupo 1 fue de 80,5 \pm 90 , y en el grupo 2 fue de $173 \pm 110$. El promedio de PCR a las $48 \mathrm{~h}$ fue de $180 \pm 113$; en el grupo 1 fue de $176,6 \pm 110$, y en el grupo 2 fue de $183,6 \pm$ 118 (Tabla 2).

El promedio de APACHE II de ingreso del total de pacientes fue de $8 \pm 4$, con valores extremos de

Tabla 1. Características generales de la cohorte

\begin{tabular}{|lcccc|}
\hline Características generales & $\begin{array}{c}\text { Grupo 1 } \\
(\mathbf{n = 3 5 )}\end{array}$ & $\begin{array}{c}\text { Grupo 2 } \\
(\mathbf{n = 3 6 )}\end{array}$ & $\begin{array}{c}\text { Total } \\
(\mathbf{n = 7 1 )}\end{array}$ & Valor p \\
\hline Edad (promedio \pm DE, años) & $56,5 \pm 18,9$ & $62,6 \pm 17,6$ & $59,6 \pm 18,4$ & 0,163 \\
Género femenino (\%) & 65 & 50 & 57 & 0,180 \\
\hline Litiásica (\%) & 94,2 & 94,4 & 94,3 & 0,682 \\
\hline
\end{tabular}

ATB: antibióticos. Grupo 1: Sin uso de ATB; Grupo 2: con uso de ATB. 
Tabla 2. Comparación de indicadores diagnósticos y pronósticos

\begin{tabular}{|lcccc|}
\hline Indicadores & $\begin{array}{c}\text { Grupo 1 } \\
(\mathbf{n = 3 5 )}\end{array}$ & $\begin{array}{c}\text { Grupo 2 } \\
(\mathbf{n = 3 6 )}\end{array}$ & $\begin{array}{c}\text { Total } \\
(\mathbf{n}=\mathbf{7 1})\end{array}$ & Valor $\mathbf{p}$ \\
\hline Amilasa de ingreso & $1.466 \pm 1.133$ & $981 \pm 896$ & $1.123 \pm 1.043$ & 0,051 \\
\hline Lipasa de ingreso & $3.393 \pm 3.578$ & $1.632 \pm 1.611$ & $2.512 \pm 2.893$ & 0,0108 \\
\hline APACHE II de ingreso & $7 \pm 4$ & $9 \pm 5$ & $8 \pm 4$ & 0,148 \\
APACHE II 48 h & $6 \pm 3$ & $9 \pm 6$ & $7 \pm 5$ & 0,033 \\
PCR de ingreso (mg/dL) & $80,5 \pm 90$ & $173 \pm 110$ & $127 \pm 110,4$ & 0,0002 \\
PCR 48 h (mg/dL) & $176,6 \pm 110$ & $183,6 \pm 118$ & $177 \pm 110,4$ & 0,8 \\
\hline
\end{tabular}

Tabla 3. Variables de desenlace de la cohorte

\begin{tabular}{|lcccc|}
\hline Variables de desenlace & $\begin{array}{c}\text { Grupo 1 } \\
(\mathbf{n = 3 5 )}\end{array}$ & $\begin{array}{c}\text { Grupo 2 } \\
(\mathbf{n = 3 6 )}\end{array}$ & $\begin{array}{c}\text { Total } \\
(\mathbf{n}=\mathbf{7 1})\end{array}$ & Valor p \\
\hline Complicaciones locales & 3 & 4 & 7 & 0,516 \\
\hline Estancia en UPC & 6 & 6 & 12 & 0,957 \\
\hline Estancia hospitalaria (promedio \pm DE, días) & $18,2 \pm 9,5$ & $22,6 \pm 29,2$ & $20,1 \pm 20,7$ & 0,6064 \\
Mortalidad & 1 & 2 & 3 & 0,511 \\
\hline
\end{tabular}

Grupo 1: Sin uso de ATB; Grupo 2: con uso de ATB.

1 y 28 ; en el grupo 1 fue de $7 \pm 4$, y en el grupo 2 fue de $9 \pm 5$. Y el promedio de APACHE II a las $48 \mathrm{~h}$ fue de $7 \pm 5$; En el grupo 1 fue de $6 \pm 3$, y en el grupo 2 fue de $9 \pm 6$ (Tabla 2).

En cuanto a las variables de desenlace (Tabla 3), la necesidad de cama en UPC fue de 12 pacientes (16\%); 6 pacientes del grupo sin antibióticos y 6 pacientes del grupo con antibióticos $(\mathrm{p}=0,957)$. Con un promedio de hospitalización en UPC del total de pacientes de 14,1 $\pm 21,1$ días; en el grupo 1 un promedio de $8 \pm 10,7$ días y en el grupo $220,2 \pm$ 28,2 días $(\mathrm{p}=0,392)$.

Siete pacientes $(9,8 \%)$ tuvieron algún tipo de complicación relacionada a la pancreatitis aguda grave, 3 pacientes correspondieron al grupo 1 y 4 al grupo $2(\mathrm{p}=0,516)$.

El promedio de hospitalización del total de pacientes fue de 20,1 $\pm 20,7$ días; en el grupo 1 fue de $18,2 \pm 9,5$ días, y en el grupo 2 fue de 22,6 $\pm 29,2$ días $(\mathrm{p}=0,495)$.

Cuarenta y nueve pacientes esperaron hospitalizados la cirugía resolutiva de su patología biliar, 4 pacientes presentaron pancreatitis alitiásica, 7 pacientes habían sido colecistectomizados previamente, por lo que se realizó colangiopancreatografía retrógrada endoscópica, 5 pacientes aún no han sido resueltos, 3 pacientes rechazaron cirugía, y 3 pacientes fallecieron previo a su resolución. El tiempo en- tre el ingreso y la cirugía resolutiva de su patología biliar $(\mathrm{n}=49)$ fue de 18,2 \pm 18,2 días, con valores extremos de 0 y 100 días; En el grupo $1(n=28)$ fue de $14,9 \pm 8$ días, y en el grupo $2(n=21)$ fue de 22,7 $\pm 25,9$ días.

Tres pacientes requirieron drenajes percutáneos, siendo insuficiente en 2 de ellos por lo que fue necesario realizar drenaje quirúrgico.

En cuanto a mortalidad, hubo 3 pacientes fallecidos $(4,23 \%)$ en todo el estudio, 1 paciente correspondió al grupo que no usó antibióticos y 2 pacientes al grupo que usó antibióticos $(\mathrm{p}=0,511)$.

\section{Discusión}

La mortalidad en pancreatitis aguda grave está claramente asociada a las complicaciones infecciosas, es por esto que la administración de antibióticos profilácticos ha sido parte del manejo de PAG por décadas. Sin embargo, la controversia continúa debido a la evidencia insuficiente ${ }^{3,4}$.

La evolución de la pancreatitis plantea que la necrosis inicial sufre licuefacción en el tiempo y luego de esto se puede reabsorber y formar una colección postnecrótica. Es por esto que desde el punto de vista teórico, si logramos disminuir la infección de la necrosis, podemos tener menos complicaciones 
locales y menor mortalidad. Hay clara evidencia de que los pacientes con complicaciones locales presentan una tasa de morbilidad y mortalidad mayor, en parte dada por el mayor riesgo de infección de éstas ${ }^{2,6,7}$.

Los antibióticos utilizados en profilaxis de PAG deben cumplir dos condiciones: cubrir las bacterias más comunes involucradas en la infección de necrosis y complicaciones locales de pacientes con PAG, y penetrar adecuadamente al tejido pancreático. En cuanto a los gérmenes más comúnmente involucrados tenemos bacterias gram negativas y anaerobias, como Escherichia coli, Klebsiella pneumoniae, Proteus y Bacteroides ${ }^{2,3}$.

En nuestro hospital, en los últimos años, se han hecho cambios en el tratamiento de la PAG, disminuyendo las complicaciones locales, sistémicas y la mortalidad. Dentro de estas medidas está el uso de antibióticos profilácticos. Sin embargo, no se tiene claro su verdadero rol ${ }^{13}$.

Sólo existe un ECA que demostró la utilidad de ATB profilácticos con carbapenémicos en pacientes con $\mathrm{PAG}^{5}$.

Dentro de las controversias sobre el uso de ATB profilácticos en PAG está el costo económico y el efecto de la exposición prolongada a un determinado esquema antibiótico, que puede generar presión sobre el ecosistema y aumento de bacterias resistentes a éstos. Las quinolonas han sido involucradas en la generación de bacterias resistentes por diversos mecanismos genéticos y no genéticos. Por consiguiente, una exposición prolongada (en tiempo y número de pacientes) a un esquema de profilaxis con quinolonas podría generar un aumento de bacterias multirresistentes. No sabemos si es causa o efecto, o sólo coincidencia, pero hemos observado y reportado un aumento en el número de bacterias multirresistentes en los últimos años en los cultivos de infecciones pancreáticas de pacientes con $\mathrm{PAG}^{14}$.

La pregunta lógica de por qué no usar carbapenémicos como profilaxis plantea una discusión ética y científica, ya que los carbapenémicos son la base del tratamiento de las infecciones multirresistentes, sobre todo de bacterias con beta-lactamasas de espectro expandido (BLEE), un problema en la ecología bacteriana mundial. Entonces, ¿cómo usar el único tratamiento disponible para estas bacterias como profilaxis?
El otro punto es la verdadera repercusión de un solo aspecto (ATB profilácticos) sobre una enfermedad cuya evolución es multifactorial, y sobre cuya mortalidad influyen factores como la reanimación al inicio de la enfermedad, alimentación enteral precoz, porcentaje y ubicación de la necrosis, entre otras $^{2-10}$.

Quisimos reportar los resultados de nuestra investigación al recolectar el $25 \%$ de la muestra. En la tabla de comparación de los grupos vemos que son perfectamente comparables (Tablas 1 y 2).

La comparación muestra que al completar el $25 \%$ de la muestra del ECA, el uso de ATB profilácticos en PAG no redujo las complicaciones infecciosas locales y/o sistémicas, necesidad real de ingreso a UPC, estancia hospitalaria, ni la mortalidad.

Estos resultados preliminares tendrán que comprobarse en el reporte del $50 \%$ de la muestra y el reporte final.

Si se mantiene esta tendencia, se reservará el uso de ATB solo para sospecha o confirmación de infección pancreática o extrapancreática, lo que tendrá un tremendo impacto económico, sobre todo en los hospitales públicos, y tendrá un impacto importante sobre los diferentes ecosistemas hospitalarios.

\section{Conclusiones}

Nuestro reporte preliminar demuestra que el uso de antibióticos profilácticos en PAG no reduce las complicaciones infecciosas locales y/o sistémicas, necesidad de cama en UPC, ni la mortalidad. Esta tendencia tendrá que demostrarse en reportes futuros.

\section{Responsabilidades éticas}

Protección de personas y animales. Los autores declaran que para esta investigación no se han realizado experimentos en seres humanos ni en animales.

Confidencialidad de los datos. Los autores declaran que en este artículo no aparecen datos de pacientes.

Conflictos de interés: no hay. 


\section{ARTÍ́CULO ORIGINAL}

\section{Bibliografía}

1. Bansal SS, Hodson J, Sutcliffe RS, Marudanayagam R, Muiesan P, Feroze Mirza D, et al. Performance of the revised Atlanta and determinant-based classifications for severity in acute pancreatitis. The British Journal of Surgery 2016;103:427-33.

2. Bai Y, Gao J, Zou DW, Li ZS. Prophylactic antibiotics cannot reduce infected pancreatic necrosis and mortality in acute necrotizing pancreatitis: evidence from a meta-analysis of randomized controlled trials. The American Journal of Gastroenterology 2008;103:104-10.

3. Jiang K, Huang W, Yang X, Xia Q. Present and future of prophylactic antibiotics for severe acute pancreatitis. World Journal of Gastroenterology 2012;18:279-84.

4. Rinninella E, Annetta MG, Serricchio ML, Dal Lago AA, Miggiano GAD, Mele MC. Nutritional support in acute pancreatitis: from physiopathology to practice. An evidence-based approach. Eur Rev Med Pharmacol Sci. 2017;21:421-32.

5. Beger HG, Gansauge F, Poch B, Schwarz $M$. The use of antibiotics for acute pancreatitis: is there a role? Current Infectious Disease Reports 2009;11:101-7.

6. Kylänpää L, Rakonczay Z, O'Reilly D. The clinical course of acute pancreatitis and the inflammatory mediators that drive it. International Journal of Inflammation 2012; Article ID 360685, 10 pages. doi: $10.1155 / 2012 / 360685$.

7. Sharma VK, Howden CW. Prophylactic antibiotic administration reduces sepsis and mortality in acute necrotizing pancreatitis: a metaanalysis. Pancreas United States; 2001;22:28-31.

8. Villatoro E, Larvin M, Bassi C. Antibiotic therapy for prophylaxis against infection of pancreatic necrosis in acute pancreatitis. The Cochrane Database of Systematic Reviews 2003, Issue 4. Art. No.: CD002941. DOI: 10.1002/14651858. CD002941.

9. Ukai T, Shikata S, Inoue M, Noguchi
Y, Igarashi H, Isaji S, et al. Early prophylactic antibiotics administration for acute necrotizing pancreatitis: a meta-analysis of randomized controlled trials. Journal of Hepatobiliary-Pancreatic Sciences 2015;22:316-21.

10. Mazaki T, Ishii Y, Takayama T. Metaanalysis of prophylactic antibiotic use in acute necrotizing pancreatitis. British Journal of Surgery 2006;93:674-84.

11. Jiang K, Huang W, Yang X-N, Xia Q. Present and future of prophylactic antibiotics for severe acute pancreatitis. World Journal of Gastroenterology 2012;18:279-84.

12. Losada MC, Burgos 1, Silva J. Protocolo de tratamiento y resultados de pancreatitis aguda: Estudio de cohorte. Rev Chil Cir. 2010;62:557-63.

13. Losada H, Burgos L, Silva J, Fernández O, Durán R, Henríquez E. Cambio en la microbiología de los abscesos pancreáticos en el Hospital Regional de Temuco. Serie de casos. Rev Chil Cir. 2006;58:20-4. 